TRANSACTIONS OF THE

AMERICAN MATHEMATICAL SOCIETY

Volume 356, Number 3, Pages 939-951

S 0002-9947(03)03454-8

Article electronically published on October 8, 2003

\title{
LOW-DEGREE POINTS ON HURWITZ-KLEIN CURVES
}

\author{
PAVLOS TZERMIAS
}

\begin{abstract}
We investigate low-degree points on the Fermat curve of degree 13, the Snyder quintic curve and the Klein quartic curve. We compute all quadratic points on these curves and use Coleman's effective Chabauty method to obtain bounds for the number of cubic points on each of the former two curves.
\end{abstract}

\section{INTRODUCTION}

Let $\mathbb{Q}$ be the field of rational numbers and let $\overline{\mathbb{Q}}$ be a fixed algebraic closure of $\mathbb{Q}$. For a curve $C$ of genus $g \geq 2$ defined over $\mathbb{Q}$, let $J$ denote the Jacobian of $C$. Assuming that $C$ has a $\mathbb{Q}$-rational point $P_{0}$, we fix an Albanese embedding of $C$ in $J$ by choosing $P_{0}$ as a base point. For a positive integer $d$, let $W_{d}$ denote the image of the $d$-th symmetric power $C^{(d)}$ of $C$ in $J$ under the map induced by the fixed Albanese embedding of $C$ in $J$. Recall that the gonality $\gamma$ of $C$ is defined as the smallest degree of a morphism from $C$ to $\mathbb{P}^{1}$. Let us recall the following special case of a celebrated theorem of Faltings ([7]):

Theorem 1.1 (Faltings). If $d<\gamma$ and $W_{d}$ does not contain a translate of a nontrivial abelian subvariety of $J$, then there are only finitely many $\overline{\mathbb{Q}}$-points on $C$ whose field of definition has degree at most $d$ over $\mathbb{Q}$.

We refer to such points as low-degree points on $C$. In the case of a smooth plane curve $C$ of degree $N \geq 7$, Debarre and Klassen (5]) have shown that the conclusion of the above theorem holds for $d \leq N-2$. Explicit versions of such results for specific curves are hard to obtain. In this paper, we will be concerned with two special types of curves, namely the Fermat curves and the Hurwitz-Klein curves whose definition we now recall:

Let $p$ be a fixed prime, such that $p \geq 5$. We denote by $F_{p}$ the Fermat curve of degree $p$, i.e. the complete plane curve over $\mathbb{Q}$ given by the projective equation

$$
X^{p}+Y^{p}+Z^{p}=0 .
$$

Suppose that, in addition, $p \equiv 1 \bmod 3$. Let $F_{p, r}$ denote a smooth projective model of the singular affine curve

$$
y^{p}=x^{r}(1-x)
$$

where $r$ is a primitive cube root of unity $\bmod p$. It was observed by Lefschetz ([13]) that $F_{p, r}$ has a cubic automorphism $\rho$. Note that $F_{p, r}$ arises as a quotient of $F_{p}$

Received by the editors January 31, 2001 and, in revised form, August 1, 2001 and May 31, 2002.

2000 Mathematics Subject Classification. Primary 11G30, 14H25; Secondary 11G10, 14 G05.

Key words and phrases. Hurwitz-Klein curves, Fermat curves, low-degree points. 
by a certain group of automorphisms of $F_{p}$ (see [8]). Also the Jacobian $J_{p, r}$ of $F_{p, r}$ has complex multiplication by a primitive $p$-th root of unity $\zeta$ in $\overline{\mathbb{Q}}$. Let $K$ denote the cyclotomic field $\mathbb{Q}(\zeta)$. It is well known from the work of Hurwitz $(9])$ that $F_{p, r}$ is also given by the (singular) model

$$
x^{m} y^{n}+y^{m}+x^{n}=0,
$$

where $m$ and $n$ are positive integers such that $m^{2}-m n+n^{2}=p$ (the existence of such integers follows from the fact that $p$ splits completely in $\mathbb{Q}(\sqrt{-3})$ ). In this model, the cubic automorphism of $F_{p, r}$ is given by $\rho(x, y)=(1 / y, x / y)$. Both models of $F_{p, r}$ will be used in this paper. We will refer to $F_{p, r}$ as a Hurwitz-Klein curve. Two of these curves, namely $F_{7,2}$ (the Klein quartic) and $F_{13,3}$ (the Snyder quintic), will be extensively studied in this paper.

While all $\mathbb{Q}$-rational points on $F_{p}$ are now known thanks to Wiles and Taylor ([21], 18]), analogous results for low-degree points on $F_{p}$ have only been obtained in special cases. Gross and Rohrlich ([8]) computed all points of degree at most $(p-1) / 2$ on $F_{p}$ for $p \leq 11$. Debarre and Klassen $([5)$ asked whether all points of degree at most $p-2$ on $F_{p}$ lie on the line $X+Y+Z=0$ (for all $p \geq 5$ ). A similar question has been raised by Ribenboim ([17]). The former question has been settled affirmatively for $p=5$ (10]) and for $p=7$ ([19]). The points of degree at most 5 on $F_{11}$ have been computed by Gross and Rohrlich in [8. Also the main result of [20] is that there are at most 120 points of degree 6 on $F_{11}$. A closer look at the latter results and their proofs leads to the following observation: in all the known cases, the $\operatorname{Gal}(\overline{\mathbb{Q}} / \mathbb{Q})$-orbit of a low-degree point $R$ on $F_{p}$ is contained in the $S_{3}$-orbit of $R$, where we identify $S_{3}$ with the obvious subgroup of the automorphism group of $F_{p}$. In this paper we verify part of the same assertion for $p=13$. Our main result is as follows:

Theorem 1.2. There are exactly 3 rational points, 2 quadratic points and at most 27 cubic points on $F_{13}$. Moreover, the Galois orbit of any of these points is contained in its $S_{3}$-orbit.

We of course claim no novelty for the result about rational points; we have included it in the statement of the above theorem only for the sake of completeness. To our knowledge, the result about quadratic and cubic points is the first example of a result of this type for a Fermat curve whose Jacobian conjecturally has no Faddeev factor (i.e. a factor of the form $J_{p, r}$ ) with finite Mordell-Weil group (the predictions of the Birch and Swinnerton-Dyer conjecture regarding Mordell-Weil ranks of Fermat Jacobians are discussed in [8]). The proof of Theorem 1.2 uses the geometry of $J_{13,3}$ (studied by Lim in [14]), Coleman's effective Chabauty bound ([2]) and a technical device (Theorem 1.3 below) which is valid only under a strong (and difficult to settle in general) Mordell-Weil rank condition. Following Lim ([14]), let $C_{0}$ denote the quotient of $F_{p, r}$ by the group of automorphisms generated by $\rho$. Let $J_{0}$ denote the Jacobian of $C_{0}$. Note that the Mordell-Weil ranks of $J_{0}$ and $J_{p, r}$ over $\mathbb{Q}$ satisfy $\operatorname{rk}\left(J_{0}(\mathbb{Q})\right) \leq \operatorname{rk}\left(J_{p, r}(\mathbb{Q})\right)$. The technical device mentioned above is the following theorem:

Theorem 1.3. Suppose that $\operatorname{rk}\left(J_{0}(\mathbb{Q})\right) \geq \operatorname{rk}\left(J_{p, r}(\mathbb{Q})\right)$ - 1 . Then there are exactly 3 rational points and 2 quadratic points on $F_{p, r}$. In particular, if the above rank condition is satisfied, there are exactly 3 rational points and 2 quadratic points on $F_{p}$. 
The fact that $F_{p, r}$ has exactly 3 rational points for all $p$ and $r$ (unconditionally) follows from the work of Wiles and Taylor ([21], [18]). We should therefore point out that we include a statement about rational points in Theorem 1.3 only for the sake of completeness. Also the Mordell-Weil rank condition in Theorem 1.3 is a strong restriction. By Corollary 2.2 in the next section, it is always satisfied if $\operatorname{rk}\left(J_{p, r}(\mathbb{Q})\right) \leq 2$ and, in particular, for $p=7$ and $p=13$. For the case $p=7$, we thus obtain a generalization of the classical theorem of Hurwitz in 9] (see also [3] for a different proof by Coleman) describing the rational points on the Klein quartic. We should also point out that the determination of the quadratic points on $F_{7,2}$ also follows from the work of Gross and Rohrlich in [8, as shown in the Ph.D. thesis of Oumar Sall. In the next section we discuss the Mordell-Weil rank condition of Theorem 1.3 in more detail (see in particular the remark following Corollary 2.2). Joint work in progress with William McCallum may provide some answers regarding the extent to which it might be true more generally.

Remark. The computation of all rational and quadratic points on the curves $F_{7,2}$ and $F_{13,3}$ easily provides similar results for curves which cover one of them over $\mathbb{Q}$. The example of Hurwitz-Klein curves of the form $X^{m} Y+Y^{m} Z+Z^{m} X=0$, where $m \geq 3$ is an integer such that $m \equiv 3 \bmod 7$ or $m \equiv 5 \bmod 7$ or $m \equiv 4 \bmod 13$ or $m \equiv 10 \bmod 13$, is discussed in section 3 (see the remark at the end of that section).

\section{The GeOMETRY of $J_{p, r}$}

Following the notation of Theorem $1.3, p$ is a prime such that $p \equiv 1 \bmod 3$ and $r$ is a primitive cube root of unity $\bmod p$. Recall that $\zeta$ is a fixed primitive $p$-th root of unity in $\overline{\mathbb{Q}}, K$ is the cyclotomic field $\mathbb{Q}(\zeta)$ and $J_{p, r}$ is the Jacobian of the Hurwitz-Klein curve $F_{p, r}$ defined in the Introduction.

Koblitz and Rohrlich ([12]) showed that $J_{p, r}$ is isogenous to a cube of an absolutely simple abelian variety. Lim (14]) explicitly computed the endomorphism ring of $J_{p, r}$ and proved that $J_{p, r}$ is $K$-isomorphic to a cube of an abelian variety. We now describe Lim's result:

For $i=0,1,2$, let $C_{i}$ denote the quotient curve of $F_{p, r}$ by the action of the automorphism group $\left\langle\zeta^{-i} \rho \zeta^{i}\right\rangle$. Let $J_{i}$ be the Jacobian of $C_{i}$. Note that $C_{0}$ is defined over $\mathbb{Q}$ and the curves $C_{i}$ are all isomorphic over $K$. Lim showed that the natural projection maps

$$
\phi_{i}: F_{p, r} \longrightarrow C_{i}
$$

give rise, by Albanese functoriality, to a $K$-isogeny

$$
\phi=\prod_{i=0}^{2} \phi_{i}: J_{p, r} \longrightarrow \prod_{i=0}^{2} J_{i}
$$

whose kernel equals $J_{p, r}[\pi]$, where $\pi=\zeta-1$. He then proceeded to show that there exists an isomorphism

$$
f=\prod_{i=0}^{2} f_{i}: J_{p, r} \longrightarrow \prod_{i=0}^{2} J_{i}
$$

defined over $K$ such that $\phi_{i}=f_{i} \pi$, for $i=0,1,2$.

For the specific case $p=7$, this result was established by Prapavessi ([16]). 
The fact that $\phi$ is only defined over $K$ makes it difficult to compare the MordellWeil ranks of $J_{p, r}$ and $J_{0}$ over $\mathbb{Q}$. Identifying $J_{0}$ and $J_{p, r}$ with their duals, it follows immediately from the definition that the dual homomorphism

$$
\left(\phi_{0}\right)^{*}: J_{0} \longrightarrow J_{p, r}
$$

satisfies the equalities

$$
\begin{gathered}
\phi_{0}\left(\phi_{0}\right)^{*}=3, \\
\left(\phi_{0}\right)^{*} \phi_{0}=1+\rho+\rho^{2} .
\end{gathered}
$$

In particular, $\left(\phi_{0}\right)^{*}$ has finite kernel and the rank of $J_{0}(\mathbb{Q})$ equals the dimension of the kernel of the endomorphism $\rho-1$ of the $\mathbb{Q}$-vector space $J_{p, r}(\mathbb{Q}) \otimes \mathbb{Q}$.

The following proposition shows in particular that the rank condition in Theorem 1.3 is equivalent to the following assertion:

$$
\operatorname{rk}\left(J_{p, r}(\mathbb{Q})\right) \stackrel{?}{=} \operatorname{rk}\left(J_{0}(\mathbb{Q})\right) .
$$

Proposition 2.1. Let $M$ be any number field. Then

$$
\operatorname{rk}\left(J_{p, r}(M)\right) \equiv \operatorname{rk}\left(J_{0}(M)\right) \bmod 2 .
$$

Proof of Proposition 2.1. We look at the isogeny decomposition of $J_{p, r}$ over $\mathbb{Q}$. Clearly, $J_{0}$ is a factor. Consider the abelian subvariety $B=(\rho-1) J_{p, r}$ of $J_{p, r}$. It is easy to show that the map

$$
J_{p, r} \stackrel{\phi_{0} \times(\rho-1)}{\longrightarrow} J_{0} \times B
$$

is an isogeny defined over $\mathbb{Q}$. Hence, it suffices to show that $\operatorname{rk}(B(M))$ is even. The automorphism $\rho$ of $J_{p, r}$ induces an endomorphism $\theta$ of $B$ such that $\theta^{2}+\theta+1=0$. Since the polynomial $X^{2}+X+1$ is irreducible over $\mathbb{Q}$, the elementary divisors of $\theta$ acting on the $\mathbb{Q}$-vector space $B(M) \otimes \mathbb{Q}$ are all equal to $X^{2}+X+1$. In particular, $B(M) \otimes \mathbb{Q}$ is a direct sum of two-dimensional cyclic $\theta$-invariant subspaces, and this completes the proof.

Corollary 2.2. If $\operatorname{rk}\left(J_{p, r}(\mathbb{Q})\right) \leq 2$, then the Mordell-Weil rank condition in Theorem 1.3 is satisfied.

Remark. It remains an open problem whether the inequality $\operatorname{rk}\left(J_{p, r}(\mathbb{Q})\right) \leq 2$ holds in general. One possible approach is to perform a $(\zeta-1)$-descent on $J_{p, r}$ using its isogeny decomposition over $K$ combined with results of Faddeev ( 6 ) and McCallum (15]). For some time, the author was under the impression that the information on the corresponding Selmer and Shafarevich-Tate groups obtained by this approach produces examples where the inequality $\operatorname{rk}\left(J_{p, r}(\mathbb{Q})\right) \leq 2$ fails. It turns out that this is not the case. At present, no counterexample to the latter inequality is known. We hope to address this question in joint work (in progress) with McCallum.

Proof of Corollary 2.2. By Proposition 2.1, we only need to show that we cannot have $\operatorname{rk}\left(J_{p, r}(\mathbb{Q})\right)=2$ and $\operatorname{rk}\left(J_{0}(\mathbb{Q})\right)=0$. If $p \geq 13$, this follows from the work of Gross and Rohrlich ([8]) together with the observation that the Gross-Rohrlich divisor class projects to a point of infinite order on $J_{0}$ (the Gross-Rohrlich divisor class is, up to torsion, $\rho$-invariant, therefore its image under $1+\rho+\rho^{2}$ is also of infinite order). If $p=7$, then both ranks are equal to 0 by a result of Faddeev ([6]). This proves the corollary. 
We now want to take advantage of the fact that $J_{p, r}$ and $J_{0}$ have complex multiplication by $\mathbb{Z}[\zeta]$ and $\mathbb{Z}\left[\zeta+\zeta^{r}+\zeta^{r^{2}}\right]$, respectively (note that $\rho \zeta=\zeta^{r} \rho$, so the endomorphism $\zeta+\zeta^{r}+\zeta^{r^{2}}$ of $J_{p, r}$ commutes with $\rho$, hence induces an endomorphism of $\left.J_{0}\right)$. Let $L=\mathbb{Q}\left(\zeta+\zeta^{r}+\zeta^{r^{2}}\right)$. Then $[K: L]=3$. The following result is well known. We have not been able to find a reference, so we give a proof of it below.

Lemma 2.3. We have the following equalities:

(a) $\operatorname{rk}\left(J_{p, r}(K)\right)=(p-1) \operatorname{rk}\left(J_{p, r}(\mathbb{Q})\right)$.

(b) $\operatorname{rk}\left(J_{p, r}(L)\right)=(p-1) \operatorname{rk}\left(J_{p, r}(\mathbb{Q})\right) / 3$.

(c) $\operatorname{rk}\left(J_{0}(L)\right)=(p-1) \operatorname{rk}\left(J_{0}(\mathbb{Q})\right) / 3$.

Proof of Lemma 2.3. We only prove part (a). The proofs of the remaining parts are similar. Let $G=\operatorname{Gal}(K / \mathbb{Q})$. Since $\zeta$ acts as complex multiplication on $J_{p, r}(K)$, the $\mathbb{Q}$-vector space $V=J_{p, r}(K) \otimes \mathbb{Q}$ has a $K$-vector space structure. With respect to this structure, the Galois action of $G$ on $V$ is semi-linear. Choosing a $K$-basis of $V$, we can thus interpret the action of $G$ as an element of the Galois cohomology group $H^{1}\left(G, G L_{n}(K)\right)$, where $n=\operatorname{dim}_{K} V$. By Hilbert's Theorem 90, the latter group is trivial, therefore $V$ is isomorphic to $V^{G} \otimes K$. In particular,

$$
\operatorname{rk}\left(J_{p, r}(K)\right)=\operatorname{dim}_{\mathbb{Q}} V=\left(\operatorname{dim}_{\mathbb{Q}} V^{G}\right)\left(\operatorname{dim}_{\mathbb{Q}} K\right)=(p-1) \operatorname{rk}\left(J_{p, r}(\mathbb{Q})\right) .
$$

Using Proposition 2.1, Lemma 2.3 and Lim's results on the geometry of $J_{p, r}$ over $K$, one sees that the rank condition in Theorem 1.3 is reduced to any of the following equivalent assertions:

$$
\begin{gathered}
\operatorname{rk}\left(\phi_{0}\left(J_{p, r}(L)\right)\right) \stackrel{?}{=} \operatorname{rk}\left(J_{p, r}(L)\right), \\
\operatorname{rk}\left(f_{0}\left((\zeta-1) J_{p, r}(L)\right)\right) \stackrel{?}{=} \operatorname{rk}\left(J_{p, r}(L)\right) .
\end{gathered}
$$

We can only prove the following statement, which, nevertheless, will be used in the proof of Theorem 1.3:

Lemma 2.4. We have:

(a) $\operatorname{rk}\left(\phi_{0}\left(\zeta^{k} J_{p, r}(L)\right)\right)=\operatorname{rk}\left(J_{p, r}(L)\right)$, for all $k \in\{1, \cdots, p-1\}$.

(b) $\operatorname{rk}\left(f_{0}\left(\left(\zeta^{k}-1\right) J_{p, r}(L)\right)\right)=\operatorname{rk}\left(J_{p, r}(L)\right)$, for all $k \in\{2,3, \cdots, p-1\}$.

Proof of Lemma 2.4. (a) Fix $k \in\{1, \cdots, p-1\}$. Suppose that the claim is false. Then there exists a point of infinite order $D \in J(L)$ such that $\phi_{0}\left(\zeta^{k} D\right)=0$. Therefore $\left(1+\rho+\rho^{2}\right)\left(\zeta^{k} D\right)=0$. Consider the abelian group

$$
G=\left\{\zeta^{k} D_{1}+\zeta^{k r} D_{2}+\zeta^{k r^{2}} D_{3}: D_{i} \in J_{p, r}(L), i=1,2,3\right\} .
$$

Since $J_{p, r}$ is $K$-isomorphic to $J_{0}^{3}$, we have $3 \operatorname{rk}\left(J_{0}(K)\right)=\operatorname{rk}\left(J_{p, r}(K)\right)$ and, by Lemma $2.3, \operatorname{rk}\left(K \operatorname{Ker}\left(\phi_{0}\right)\right)=2 \operatorname{rk}\left(J_{p, r}(L)\right)$. Note that, by the proof of Lemma 2.3, we have $G \otimes \mathbb{Q}=J_{p, r}(K) \otimes \mathbb{Q}$. Therefore, $\operatorname{rk}\left(\operatorname{Ker}\left(\phi_{0}\right) \cap G\right)=2 \operatorname{rk}\left(J_{p, r}(L)\right)$. Now consider the following subgroup $H$ of $G$ :

$$
H=\left\{\zeta^{k} D_{1}+\zeta^{k r} D_{2}+\zeta^{k r^{2}} D_{3}: D_{1}+D_{2}^{\rho^{2}}+D_{3}^{\rho}=\lambda D, \lambda \in \mathbb{Z}\right\} .
$$

$H$ has rank strictly greater than $2 \operatorname{rk}\left(J_{p, r}(L)\right)$, since we can choose $D_{2}$ and $D_{3}$ to be any points of infinite order in $J_{p, r}(L)$ and we still have infinitely many choices for $D_{1}$. However, since $\rho \zeta=\zeta^{r} \rho$, it is trivial to check that, for $F \in H,\left(1+\rho+\rho^{2}\right)(F)=$ $\left(1+\rho+\rho^{2}\right)\left(\lambda \zeta^{k} D\right)=0$. Hence, $H \subseteq \operatorname{Ker}\left(\phi_{0}\right) \cap G$, which is impossible by the above discussion regarding the ranks of these groups. 
(b) Fix $k \in\{2,3, \cdots, p-1\}$. Set

$$
\eta_{i}=\sum_{j=0}^{k-1} \zeta^{j r^{i}},
$$

for $i=0,1,2$. Since $\phi_{0}=f_{0} \pi$, it suffices to show that there is no point $D$ of infinite order in $J_{p, r}(L)$ such that $\left(1+\rho+\rho^{2}\right)\left(\eta_{0} D\right)=0$. Suppose this were not the case. Applying $\operatorname{Gal}(K / L)$ to the latter equality we get the system

$$
\begin{aligned}
& \eta_{0} D+\eta_{1} D^{\rho}+\eta_{2} D^{\rho^{2}}=0, \\
& \eta_{1} D+\eta_{2} D^{\rho}+\eta_{0} D^{\rho^{2}}=0, \\
& \eta_{2} D+\eta_{0} D^{\rho}+\eta_{1} D^{\rho^{2}}=0 .
\end{aligned}
$$

Eliminating $D^{\rho}$ and $D^{\rho^{2}}$ from the above system, we get

$$
\left(\left(\eta_{0}^{2}-\eta_{1} \eta_{2}\right)^{2}-\left(\eta_{1}^{2}-\eta_{0} \eta_{2}\right)\left(\eta_{2}^{2}-\eta_{0} \eta_{1}\right)\right) D=0 .
$$

It is easy to check that the coefficient of $D$ is a non-zero element in $\mathbb{Z}[\zeta]$. Multiplying the latter equality by an appropriate integer in $\mathbb{Z}[\zeta]$, we obtain $s D=0$, for a nonzero integer $s \in \mathbb{Z}$ (we can take $s$ to be the $K / \mathbb{Q}$-norm of the original coefficient). This implies that $D$ is torsion, which is a contradiction.

\section{Algebraic points of Low Degree}

In this section we will prove Theorem 1.3. Consider the cusps on (the first model of) $F_{p, r}$ :

$$
a=(0,0), \quad b=(1,0), \quad c=\infty .
$$

Note that $\rho$ induces a permutation of $\{a, b, \infty\}$ of order 3 and $\zeta$ fixes each of these three points. By the work of Gross and Rohrlich ([8]), the difference between any two cusps is a $p$-torsion point on $J_{p, r}$. For a point $P_{1}$ on $F_{p, r}$ of degree $k$ over $\mathbb{Q}$, let $P_{1}, \cdots, P_{k}$ be the Galois conjugates of $P_{1}$. Let $D$ be the following point in $J_{p, r}(\mathbb{Q})$ :

$$
D=\left[P_{1}+\cdots+P_{k}-k \infty\right] .
$$

Lemma 3.1. Let $p \geq 13$. Suppose that $P_{1} \neq a, b, \infty$.

(a) If $k \leq 2$, then $D$ is of infinite order.

(b) If $k=3$ and the gonality $\gamma$ of $F_{p, r}$ satisfies $\gamma \geq 4$, then $D$ is of infinite order.

Proof of Lemma 3.1. Suppose that $D$ is torsion. By the work of Gross and Rohrlich ([8]), $D$ has to be invariant under $\zeta$. In other words, the divisor

$$
P_{1}+\cdots+P_{k}-\left(\zeta P_{1}+\cdots+\zeta P_{k}\right)
$$

is principal.

(a) By a result of Coleman ([3]), $F_{p, r}$ is not hyperelliptic, hence $\gamma \geq 3$. Therefore, since $k \leq 2$, the latter divisor is equal to 0 . If $k=1$, this amounts to $P_{1}=\zeta P_{1}$, which is absurd, since the left-hand side is $\mathbb{Q}$-rational, while the right-hand side is not. Similarly, if $k=2$, the only possibility is

$$
P_{1}=\zeta P_{2}, \quad P_{2}=\zeta P_{1} .
$$

But then $P_{1}=\zeta^{2} P_{1}$. Using the first model of $F_{p, r}$, this amounts to $(x, y)=\left(x, \zeta^{2} y\right)$, so $y=0$, hence $P_{1}$ is a cusp, which is a contradiction. 
(b) By the gonality assumption, we have

$$
\sum_{i=1}^{3} P_{i}=\sum_{i=1}^{3} \zeta P_{i} .
$$

As in part (a), we cannot have $P_{i}=\zeta P_{i}$, for any $i$. Therefore, $P_{1}=\zeta P_{2}, P_{2}=\zeta P_{3}$ and $P_{3}=\zeta P_{1}$ or $P_{1}=\zeta P_{3}, P_{2}=\zeta P_{1}$ and $P_{3}=\zeta P_{2}$. In either of these cases, $P_{1}=\zeta^{3} P_{1}$, which is absurd, as in part (a).

Proof of Theorem 1.3 for $p \geq 13$. Assume that the Mordell-Weil rank condition in Theorem 1.3 is satisfied. By Proposition 2.1 , we have $\operatorname{rk}\left(J_{0}(\mathbb{Q})\right)=\operatorname{rk}\left(J_{p, r}(\mathbb{Q})\right)$. By the discussion preceding Proposition 2.1, this means that $D^{\rho}-D$ is torsion, for every $D \in J_{p, r}(\mathbb{Q})$.

Now let $P_{1}$ be a point on $F_{p, r}$ of degree $k$ over $\mathbb{Q}$ (where $k=1$ or 2 ). Suppose that $P_{1} \neq a, b, \infty$. Form the corresponding $\mathbb{Q}$-rational point $D$ in $J_{p, r}(\mathbb{Q})$, as in Lemma 3.1. It follows from Lemma 3.1 that $D$ is of infinite order. Also, by the preceding paragraph, $D^{\rho}-D$ is torsion. Since the difference between any two cusps is also torsion, we get that

$$
\left[P_{1}+\cdots+P_{k}-P_{1}^{\rho}-\cdots-P_{k}^{\rho}\right]
$$

is torsion. We now distinguish two cases:

Case 1: Suppose that $p \geq 19$. By [8], the latter divisor class is invariant by $\zeta$. In other words, there exists a rational function on $F_{p, r}$ whose divisor equals

$$
P_{1}+\cdots+P_{k}+\zeta P_{1}^{\rho}+\cdots+\zeta P_{k}^{\rho}-\left(P_{1}^{\rho}+\cdots+P_{k}^{\rho}+\zeta P_{1}+\cdots+\zeta P_{k}\right) .
$$

If $k=1$, then, since the gonality $\gamma$ of $F_{p, r}$ satisfies $\gamma \geq 3$, we get that

$$
P_{1}+\zeta P_{1}^{\rho}=P_{1}^{\rho}+\zeta P_{1} .
$$

We cannot have $P_{1}=\zeta P_{1}$, because the left-hand side is defined over $\mathbb{Q}$, while the right-hand side is not. Hence, $P_{1}=P_{1}^{\rho}$. In terms of coordinates (using the second model of $F_{p, r}$ ), this means $x y=1$ and $x=y^{2}$. Hence, since $x, y \in \mathbb{Q}$, we get $x=y=1$, which is impossible because $(1,1)$ is not a point on $F_{p, r}$.

Now suppose that $k=2$. Since $\phi_{0}\left(P_{i}\right)=\phi_{0}\left(P_{i}^{\rho}\right)$, we get that the divisor

$$
\phi_{0}\left(\zeta P_{1}^{\rho}\right)+\phi_{0}\left(\zeta P_{2}^{\rho}\right)-\phi_{0}\left(\zeta P_{1}\right)-\phi_{0}\left(\zeta P_{2}\right)
$$

on $C_{0}$ is principal. Let $E$ be the divisor $\phi_{0}\left(\zeta P_{1}\right)+\phi_{0}\left(\zeta P_{2}\right)$. Since $p \geq 19$, the genus of $C_{0}$ (which equals $(p-1) / 6$ ) is at least 3 . Hence, by Riemann-Roch (notation as in [1]), we get $l(E) \leq l(K-E)$, so $E$ is a special divisor. By Clifford's theorem, $\operatorname{dim}|E|=0$ unless $C_{0}$ is hyperelliptic and $|E|$ equals the unique $g_{2}^{1}$ on $C_{0}$.

In the latter case, the points $\phi_{0}\left(\zeta P_{1}\right)$ and $\phi_{0}\left(\zeta P_{2}\right)$ are conjugate under the hyperelliptic involution of $C_{0}$. Since the latter involution acts as multiplication by -1 on $J_{0}$, we see that

$$
\left[\phi_{0}\left(\zeta P_{1}\right)+\phi_{0}\left(\zeta P_{2}\right)-2 \phi_{0}(\infty)\right]=0 .
$$

In other words, the divisor class $\zeta\left[P_{1}+P_{2}-2 \infty\right] \in \zeta J_{p, r}(\mathbb{Q})$ projects to 0 under $\phi_{0}$. By Lemma 2.4(a), this means that $\left[P_{1}+P_{2}-2 \infty\right]$ is torsion, which is impossible, by Lemma 3.1 .

Therefore, we must have $\operatorname{dim}|E|=0$. This implies that

$$
\phi_{0}\left(\zeta P_{1}^{\rho}\right)+\phi_{0}\left(\zeta P_{2}^{\rho}\right)=\phi_{0}\left(\zeta P_{1}\right)+\phi_{0}\left(\zeta P_{2}\right) .
$$


If $\phi_{0}\left(\zeta P_{1}^{\rho}\right)=\phi_{0}\left(\zeta P_{1}\right)$, then we have the following possibilities:

(i) $\zeta P_{1}^{\rho}=\zeta P_{1}$. Then $P_{1}^{\rho}=P_{1}$, so, in terms of the second model of $F_{p, r}$, we have $x y=1$ and $x=y^{2}$. This implies that $(x, y)=\left(\eta, \eta^{2}\right)$ or $\left(\eta^{2}, \eta\right)$, where $\eta$ is a primitive cube root of unity in $\overline{\mathbb{Q}}$. So we have recovered the Gross-Rohrlich points (8]).

(ii) $\zeta P_{1}^{\rho}=\zeta^{r} P_{1}^{\rho}$ or $\zeta^{r^{2}} P_{1}^{\rho^{2}}$. Then $P_{1}^{\rho}=\zeta^{r-1} P_{1}^{\rho}$ or $\zeta^{r^{2}-1} P_{1}^{\rho^{2}}$. This is impossible, since the left-hand side is defined over a quadratic field, while the right-hand side is not.

The only other option is $\phi_{0}\left(\zeta P_{1}^{\rho}\right)=\phi_{0}\left(\zeta P_{2}\right)$ and $\phi_{0}\left(\zeta P_{2}^{\rho}\right)=\phi_{0}\left(\zeta P_{1}\right)$. As above, for rationality reasons, we cannot have $\zeta P_{1}^{\rho}=\zeta^{r} P_{2}^{\rho}$ or $\zeta^{r^{2}} P_{2}^{\rho^{2}}$. Therefore, we must have $\zeta P_{1}^{\rho}=\zeta P_{2}$ and, similarly, $\zeta P_{2}^{\rho}=\zeta P_{1}$. Hence, $P_{1}^{\rho}=P_{2}$ and $P_{2}^{\rho}=P_{1}$. This shows that $P_{1}=P_{1}^{\rho^{2}}$ and, as before, $P_{1}$ is a Gross-Rohrlich point. But then $P_{1}^{\rho}=P_{1} \neq P_{2}$, a contradiction.

Case 2: Suppose that $p=13$. Note that the curve $F_{13,3}$ has the smooth plane model $x^{4} y+y^{4}+x=0$. Therefore, $\gamma=4$. The proof of Case 1 above goes through for $k=1$. For $k=2$, part of the proof goes through, up to the point where we show that there exists a rational function on $F_{p, r}$ whose divisor equals

$$
P_{1}+P_{2}+\zeta P_{1}^{\rho}+\zeta P_{2}^{\rho}-P_{1}^{\rho}-P_{2}^{\rho}-\zeta P_{1}-\zeta P_{2} \text {. }
$$

Let $E=P_{1}^{\rho}+P_{2}^{\rho}+\zeta P_{1}+\zeta P_{2}$. By Riemann-Roch, $E$ is special. By Clifford's theorem, $\operatorname{dim}|E| \leq 1$, since $E$ is not the canonical divisor (by degree) and $F_{13,3}$ is non-hyperelliptic.

Suppose $\operatorname{dim}|E|=0$. Then

$$
P_{1}+P_{2}+\zeta P_{1}^{\rho}+\zeta P_{2}^{\rho}=P_{1}^{\rho}+P_{2}^{\rho}+\zeta P_{1}+\zeta P_{2} .
$$

For rationality reasons, none of $P_{1}^{\rho}$ or $P_{2}^{\rho}$ can equal one of $\zeta P_{1}^{\rho}$ or $\zeta P_{2}^{\rho}$. Therefore, we must have $P_{1}^{\rho}+P_{2}^{\rho}=P_{1}+P_{2}$. If $P_{1}^{\rho}=P_{1}$, then, as before, we recover the Gross-Rohrlich points. If $P_{1}^{\rho}=P_{2}$, then also $P_{2}^{\rho}=P_{1}$, so $P_{1}=P_{1}^{\rho^{2}}$, and, as before, this is a contradiction.

Otherwise, if $\operatorname{dim}|E|=1$, then, by a well-known theorem in the geometry of smooth plane curves (see [1], exercise 18, page 56), there exists a line $L$ in $\mathbb{P}^{2}$ such that

$$
E=L \cdot F_{13,3}-P,
$$

where $P$ is a point on $F_{13,3}$.

Using the fact that $\left[P_{1}+P_{2}-P_{1}^{\rho}-P_{2}^{\rho}\right]$ is also invariant under $\zeta^{2}$ and arguing exactly as above, we get that for the divisor $E^{\prime}=P_{1}^{\rho}+P_{2}^{\rho}+\zeta^{2} P_{1}+\zeta^{2} P_{2}$ there exists a line $L^{\prime}$ in $\mathbb{P}^{2}$ such that

$$
E^{\prime}=L^{\prime} \cdot F_{13,3}-Q
$$

where $Q$ is a point on $F_{13,3}$ (if $\operatorname{dim}\left|E^{\prime}\right|=0$, we are done by the previous analysis).

But then $L$ and $L^{\prime}$ have the points $P_{1}^{\rho}$ and $P_{2}^{\rho}$ in common, so they must coincide. Therefore, one of the points $\zeta^{2} P_{1}$ or $\zeta^{2} P_{2}$ has to equal one of the points $\zeta P_{1}$ or $\zeta P_{2}$. As before, this is impossible for rationality reasons.

Proof of Theorem 1.3 for $p=7$. Our curve $F_{7,2}$ is the Klein curve $x^{3} y+y^{3}+x=0$. It is a smooth plane quartic, hence has gonality 3 . We will temporarily use the model $y^{7}=x(1-x)^{2}$ of $F_{7,2}$. Let us first recall that, by a result of Faddeev ([ 6 ), $J_{7,2}(\mathbb{Q})$ is finite. Also, let $P$ and $\bar{P}$ be the two quadratic points on $F_{7,2}$ that Gross 
and Rohrlich described. In fact, it follows from [8] that 2 divides the order of $[P+\bar{P}-2 \infty]$. Therefore, by $\left[8\right.$, any $\mathbb{Q}$-rational point on $J_{7,2}$ can be expressed as a $\mathbb{Z}$-linear combination of $[3 P+3 \bar{P}-2 a-2 b-2 \infty]$ and $[a-b]$. Since the first divisor class is invariant under $\rho$, it follows that for $D \in J_{7,2}(\mathbb{Q})$, we have $D^{\rho}-D \in J_{7,2}$ [7]. Following the notation in [8], we conclude that, in particular,

$$
\left[P_{1}+\cdots+P_{k}-P_{1}^{\rho}-\cdots-P_{k}^{\rho}\right]=l\left[D_{1}-D_{\infty}\right],
$$

where $0 \leq l \leq 6, D_{1}=a-b$ and $D_{\infty}=a-\infty$. From the given model of $F_{7,2}$ and the discussion in [8] (pages 203-204), it follows that

$$
3\left[D_{\infty}\right]=2\left[D_{1}\right] .
$$

Using the latter equality, we get the following cases:

If $l=6$, then $P_{1}+\cdots+P_{k}-P_{1}^{\rho}-\cdots-P_{k}^{\rho} \sim 6 D_{1}-6 D_{\infty} \sim D_{\infty}-D_{1}=b-\infty$.

If $l=5$, then $P_{1}+\cdots+P_{k}-P_{1}^{\rho}-\cdots-P_{k}^{\rho} \sim 2 D_{\infty}-2 D_{1} \sim-D_{\infty}=\infty-a$.

If $l=4$, then $P_{1}+\cdots+P_{k}-P_{1}^{\rho}-\cdots-P_{k}^{\rho} \sim 3 D_{\infty}-3 D_{1}=-D_{1}=b-a$.

If $l=3$, then $P_{1}+\cdots+P_{k}-P_{1}^{\rho}-\cdots-P_{k}^{\rho} \sim 3 D_{1}-3 D_{\infty}=D_{1}=a-b$.

If $l=2$, then $P_{1}+\cdots+P_{k}-P_{1}^{\rho}-\cdots-P_{k}^{\rho} \sim 2 D_{1}-2 D_{\infty}=D_{\infty}=a-\infty$.

If $l=1$, then $P_{1}+\cdots+P_{k}-P_{1}^{\rho}-\cdots-P_{k}^{\rho} \sim D_{1}-D_{\infty} \sim \infty-b$.

If $l=0$, then $P_{1}+\cdots+P_{k}-P_{1}^{\rho}-\cdots-P_{k}^{\rho} \sim 0$.

Now, if $k=1$, the above equivalences are equalities, because the gonality of $F_{7,2}$ equals 3 . Since $P_{1}$ is not a cusp, we reach a contradiction.

Otherwise, suppose $k=2$. We now use the smooth plane model of $F_{7,2}$. In all of the above cases, we can write

$$
P_{1}+P_{2}+Q \sim P_{1}^{\rho}+P_{2}^{\rho}+R
$$

where $R$ and $Q$ are cusps. Let $E=P_{1}+P_{2}+Q$. By Riemann-Roch and Clifford's theorem, either $\operatorname{dim}|E|=0$ or $E$ is special and $\operatorname{dim}|E|=1$.

In the former case, we get

$$
P_{1}+P_{2}+Q=P_{1}^{\rho}+P_{2}^{\rho}+R .
$$

Since $P_{1}$ is not $\mathbb{Q}$-rational, we necessarily have $Q=R$, so $P_{1}+P_{2}=P_{1}^{\rho}+P_{2}^{\rho}$, so, as before, we recover the Gross-Rohrlich points.

In the latter case, we can argue again by means of exercise 18, page 56 in [1. We can find a line $L$ in $\mathbb{P}^{2}$ and a point $Q^{\prime}$ on $F_{7,2}$ such that

$$
P_{1}+P_{2}+Q+Q^{\prime}=L \cdot F_{7,2} \text {. }
$$

Since two points determine a line and the divisor $P_{1}+P_{2}+Q$ is $\mathbb{Q}$-rational, we conclude that $L=L^{\sigma}$ for all $\sigma \in \operatorname{Gal}(\overline{\mathbb{Q}} / \mathbb{Q})$, so $L$ is a $\mathbb{Q}$-rational line, hence $Q^{\prime}$ is also a $\mathbb{Q}$-rational point.

Now if $Q=Q^{\prime}$, then $L$ is the tangent line to $F_{7,2}$ at $Q$, so an easy calculation shows that $P_{1}$ and $P_{2}$ are cusps, which is impossible since $P_{1}$ is not $\mathbb{Q}$-rational. Therefore, $Q \neq Q^{\prime}$, in which case $L$ connects two cusps. Therefore, both $P_{1}$ and $P_{2}$ have to be cusps, a contradiction.

This completes the proof of Theorem 1.3. Note that since $F_{p, r}$ is covered by $F_{p}$ by means of an explicit map (see [8]), the results automatically transfer to $F_{p}$ (a point of degree $\leq k$ on $F_{p}$ necessarily projects to a point of degree $\leq k$ on $F_{p, r}$ and knowing the latter determines the former).

Remark. The rank condition in Theorem 1.3 is satisfied for $p=7$, since Faddeev showed in [6] that $\operatorname{rk}\left(J_{7,2}(\mathbb{Q})\right)=0$. The rank condition is also satisfied for $p=13$. 
In that case, we know that $\operatorname{rk}\left(J_{13,3}(\mathbb{Q})\right)=1$, by the work of Faddeev and GrossRohrlich (see [8, second table on page 219). Therefore, by Theorem 1.3, we know all the points of degree $\leq 2$ on $F_{7,2}$ and $F_{13,3}$. We now describe how similar results can be obtained for the Hurwitz-Klein curves mentioned in the Introduction. For an integer $m \geq 3$, the curves

$$
H_{m}: x^{m} y+y^{m}+x=0, \quad G_{m}: y^{m^{2}-m+1}=x(1-x)^{m-1}
$$

are birationally isomorphic by means of the maps

$$
\begin{aligned}
H_{m} \longrightarrow G_{m}, & G_{m} \\
(x, y) \mapsto\left(\frac{-x}{y^{m}}, \frac{(-1)^{m} x}{y}\right), & (a, b) \mapsto\left(\frac{a-1}{b^{m-1}}, \frac{(-1)^{m}(a-1)}{b^{m}}\right),
\end{aligned}
$$

respectively.

(i) Suppose $m$ is of the form $7 s+3$ or $7 s+5$, with $s \in \mathbb{Z}$. Then $m^{2}-m+1=7 t$ for some integer $t$. Consider the maps $G_{m} \rightarrow G_{3}$ and $G_{m} \rightarrow G_{3}^{\prime}$ given by

$$
(x, y) \mapsto\left(x, \frac{y^{t}}{(1-x)^{s}}\right),
$$

where $G_{3}^{\prime}$ is the curve $y^{7}=x(1-x)^{4}$. Since we know all the points of degree $\leq 2$ on $F_{7,2}$ and $G_{3}, G_{3}^{\prime}$ are isomorphic to $F_{7,2}$ (see [3]), the only possible quadratic points on $H_{m}$ are the Gross-Rohrlich points which lie on $H_{m}$ if and only if 3 does not divide $m-2$.

(ii) Suppose $m$ is of the form $13 s+4$ or $13 s+10$, with $s \in \mathbb{Z}$. Then $m^{2}-m+1=13 t$ for some integer $t$. Consider the maps $G_{m} \rightarrow G_{4}$ and $G_{m} \rightarrow G_{4}^{\prime}$ given by

$$
(x, y) \mapsto\left(x, \frac{y^{t}}{(1-x)^{s}}\right),
$$

where $G_{4}^{\prime}$ is the curve $y^{13}=x(1-x)^{9}$. As in the previous case, since we know all the points of degree $\leq 2$ on $F_{13,3}$ and $G_{4}$ and $G_{4}^{\prime}$ are isomorphic to $F_{13,3}$ (see [3]), the only possible quadratic points on $H_{m}$ are the Gross-Rohrlich points which lie on $H_{m}$ if and only if 3 does not divide $m-2$.

\section{Proof of the main Result}

The statement about points of degree $\leq 2$ on $F_{13}$ follows from Theorem 1.3 , since the hypothesis of Corollary 2.2 is satisfied in this case by the results of Faddeev (see [6], paragraph 8, page 68). We will first describe all points of degree 3 on the curve $F_{13,3}$. Let $P_{1}$ be such a point. We will argue as in the proof of Theorem 1.3 for $p=7$. We use the model $y^{13}=x(1-x)^{3}$ of $F_{13,3}$. As in the latter proof, we have that

$$
\left[P_{1}+P_{2}+P_{3}-P_{1}^{\rho}-P_{2}^{\rho}-P_{3}^{\rho}\right]=l\left[D_{1}-D_{\infty}\right],
$$

where $0 \leq l \leq 12, D_{1}=a-b$ and $D_{\infty}=a-\infty$. By [8], we have

$$
4\left[D_{\infty}\right]=3\left[D_{1}\right] .
$$

We have the following cases:

$$
\begin{aligned}
& \text { If } l=12 \text {, then } P_{1}+P_{2}+P_{3}-P_{1}^{\rho}-P_{2}^{\rho}-P_{3}^{\rho} \sim D_{\infty}-D_{1}=b-\infty \text {. } \\
& \text { If } l=11 \text {, then } P_{1}+P_{2}+P_{3}-P_{1}^{\rho}-P_{2}^{\rho}-P_{3}^{\rho} \sim 2 D_{\infty}-2 D_{1}=2 b-2 \infty . \\
& \text { If } l=10 \text {, then } P_{1}+P_{2}+P_{3}-P_{1}^{\rho}-P_{2}^{\rho}-P_{3}^{\rho} \sim 3 D_{\infty}-3 D_{1}=-D_{\infty}=\infty-a \text {. } \\
& \text { If } l=9 \text {, then } P_{1}+P_{2}+P_{3}-P_{1}^{\rho}-P_{2}^{\rho}-P_{3}^{\rho} \sim-D_{1}=b-a . \\
& \text { If } l=8 \text {, then } P_{1}+P_{2}+P_{3}-P_{1}^{\rho}-P_{2}^{\rho}-P_{3}^{\rho} \sim D_{\infty}-2 D_{1}=2 b-a-\infty .
\end{aligned}
$$


If $l=7$, then $P_{1}+P_{2}+P_{3}-P_{1}^{\rho}-P_{2}^{\rho}-P_{3}^{\rho} \sim-2 D_{\infty}=2 \infty-2 a$.

If $l=6$, then $P_{1}+P_{2}+P_{3}-P_{1}^{\rho}-P_{2}^{\rho}-P_{3}^{\rho} \sim 2 D_{\infty}=2 a-2 \infty$.

If $l=5$, then $P_{1}+P_{2}+P_{3}-P_{1}^{\rho}-P_{2}^{\rho}-P_{3}^{\rho} \sim 2 D_{1}-D_{\infty}=a+\infty-2 b$.

If $l=4$, then $P_{1}+P_{2}+P_{3}-P_{1}^{\rho}-P_{2}^{\rho}-P_{3}^{\rho} \sim D_{1}=a-b$.

If $l=3$, then $P_{1}+P_{2}+P_{3}-P_{1}^{\rho}-P_{2}^{\rho}-P_{3}^{\rho} \sim D_{\infty}=a-\infty$.

If $l=2$, then $P_{1}+P_{2}+P_{3}-P_{1}^{\rho}-P_{2}^{\rho}-P_{3}^{\rho} \sim 2 D_{1}-2 D_{\infty}=2 \infty-2 b$.

If $l=1$, then $P_{1}+P_{2}+P_{3}-P_{1}^{\rho}-P_{2}^{\rho}-P_{3}^{\rho} \sim D_{1}-D_{\infty}=\infty-b$.

If $l=0$, then $P_{1}+P_{2}+P_{3}-P_{1}^{\rho}-P_{2}^{\rho}-P_{3}^{\rho} \sim 0$.

In the last case $(l=0)$, the equivalence must be an equality because the gonality of $F_{13,3}$ equals 4 . We cannot have $P_{1}=P_{1}^{\rho}$ because then, as before, $P_{1}$ would be one of the Gross-Rohrlich points. Hence, the Galois conjugates of $P_{1}$ are given by the action of $\langle\rho\rangle$ on $P_{1}$.

In each of the remaining 12 cases $(l \neq 0)$, we can add (if necessary) a cusp to both sides of the equivalence to get

$$
P_{1}+P_{2}+P_{3}+2 R \sim P_{1}^{\rho}+P_{2}^{\rho}+P_{3}^{\rho}+R^{\prime}+R^{\prime \prime},
$$

where $R, R^{\prime}$ and $R^{\prime \prime}$ are cusps. Now let $E=P_{1}+P_{2}+P_{3}+2 R$. By Riemann-Roch, $l(E)=l(K-E)$, so $E$ is special. By Clifford's theorem, $\operatorname{dim}|E| \leq 2$.

If $\operatorname{dim}|E|=0$, we conclude, as above, that the Galois conjugates of $P_{1}$ are given by the action of $\langle\rho\rangle$ on $P_{1}$.

If $\operatorname{dim}|E|=2$, then, by [1, $|E|$ is the unique $g_{5}^{2}$ on $F_{13,3}$ and is cut out by lines in $\mathbb{P}^{2}$ (recall that $F_{13,3}$ is a smooth plane quintic). In particular, there exists a line $L$ in $\mathbb{P}^{2}$ such that $E=L . F_{13,3}$. If $\operatorname{dim}|E|=1$, then, by a result of Coppens (4]), there exists a line $L$ in $\mathbb{P}^{2}$ such that $E=L . F_{13,3}$. In both cases, the line $L$ is therefore the tangent line to $F_{13,3}$ at $R$. Since $R$ is a cusp, we conclude that $P_{1}$, $P_{2}$ and $P_{3}$ are also cusps, which is impossible.

We have thus proved that the Galois orbit of a cubic point on $F_{13,3}$ is contained in its $\langle\rho\rangle$-orbit. It turns out that we get cubic points on $F_{13,3}$ by intersecting the smooth plane model of the curve with the line $x+y+1=0$ or with the conic $x y+x+y=0$. Hence there are at least 6 cubic points on $F_{13,3}$. We now want to give an upper bound for the number of such points. This can be done as follows:

Let $P_{1}$ be a cubic point on $F_{13,3}$. Consider the projection map

$$
F_{13,3} \stackrel{\phi_{0}}{\longrightarrow} C_{0} \text {. }
$$

Since Galois conjugation acts by $\langle\rho\rangle$ on $P_{1}$, it is immediate that $\phi_{0}\left(P_{1}\right)$ is $\mathbb{Q}$-rational. Since $C_{0}$ has genus 2 and Mordell-Weil rank 1 over $\mathbb{Q}$, we can apply Coleman's effective Chabauty ([2]): $C_{0}$ has good reduction at 3, therefore, by [2], Corollary $4 \mathrm{~b}(\mathrm{i})$, we get $\# C(\mathbb{Q}) \leq 12$. One of the $\mathbb{Q}$-rational points on $C_{0}$ is the projection of a cusp on $F_{13,3}$, so it must be discarded. Therefore there are at most 11 remaining $\mathbb{Q}$-rational points and they can be obtained by at most 33 cubic points on $F_{13,3}$, and this is our upper bound.

Now let us see how to transfer the above information to cubic points on $F_{13}$. First note that the map

$$
F_{13} \stackrel{g}{\longrightarrow} F_{13,3}
$$

given by

$$
(x, y) \mapsto\left(-x^{13}, x^{3} y\right)
$$

(using the first model of $F_{13,3}$ ) is injective on cubic points, because if for two cubic points $(a, b)$ and $(c, d)$ on $F_{13}$ we have $g(a, b)=g(c, d)$, then $a / c=\zeta^{l}$ for some $l$. 
If 13 does not divide $l$, we have a contradiction, since the field of definition of $a / c$ cannot exceed 9. Therefore, $a=c$, so $b=d$ also.

Also the six known cubic points on $F_{13,3}$ (obtained by intersecting the smooth plane model of $F_{13,3}$ with the line $x+y+1=0$ or with the conic $x y+x+y=0$ ) do not lift to cubic points on $F_{13}$. Hence there are at most 27 cubic points on $F_{13}$. The assertion about their Galois orbits is established as follows:

Let $P_{1}=(x, y)$ be a cubic point on $F_{13}$. Consider the automorphism (also denoted by $\rho)$ of $F_{13}$ given by $\rho(x, y)=(1 / y, x / y)$. By what we saw above we have

$$
g\left(P_{1}\right)+g\left(P_{2}\right)+g\left(P_{3}\right)=g\left(P_{1}\right)^{\rho}+g\left(P_{1}\right)^{\rho}+g\left(P_{1}\right)^{\rho} .
$$

Let $\sigma$ and $\tau$ be the non-trivial embeddings of the field of definition of $P_{1}$ in $\overline{\mathbb{Q}}$. Using the first model of $F_{13,3}$, the automorphism $\rho$ is given by

$$
(x, y) \mapsto\left(1 /(1-x),-x / y^{4}\right) .
$$

Therefore,

$$
\begin{aligned}
& \left(-x^{13}, x^{3} y\right)+\left(-\left(x^{\sigma}\right)^{13},\left(x^{\sigma}\right)^{3} y^{\sigma}\right)+\left(-\left(x^{\tau}\right)^{13},\left(x^{\tau}\right)^{3} y^{\tau}\right) \\
& =\left(-x^{13}, x^{3} y\right)+\left(-1 / y^{13}, x / y^{4}\right)+\left(-y^{13} / x^{13}, y^{3} / x^{4}\right) .
\end{aligned}
$$

Without loss of generality, we may assume that the second summands of the leftand right-hand side are equal. Then $\left(x^{\sigma} y\right)^{13}=1$ and $\left(x^{\sigma}\right)^{3} y^{4} y^{\sigma}=x$. But $x^{\sigma} y$ lies in the Galois closure of the field of definition of $P_{1}$, hence has degree at most 6 over $\mathbb{Q}$. Therefore, we get $x^{\sigma} y=1$ and $y y^{\sigma}=x$. In other words,

$$
\left(x^{\sigma}, y^{\sigma}\right)=(1 / y, x / y)=\rho(x, y)
$$

and the assertion is proved.

\section{ACKNOWLEDGMENTS}

I am grateful to William McCallum for sharing with me his insights on the subject via numerous conversations. I thank Robert Coleman, Minhyong Kim, Fernando Rodriguez Villegas and David Rohrlich for their remarks. The constructive criticism of an anonymous referee regarding the exposition is also greatly appreciated.

\section{REFERENCES}

[1] E. Arbarello, M. Cornalba, P. Griffiths and J. Harris: Geometry of algebraic curves I, Grundlehren der Math. Wiss. 247, Springer-Verlag, New York, 1985. MR 86h:14019

[2] R. Coleman: Effective Chabauty, Duke Math. J. 52 (1985), no. 3, 765-770. MR 87f:11043

[3] R. Coleman: Torsion points on abelian étale coverings of $\mathbb{P}^{1}-\{0,1, \infty\}$, Trans. Amer. Math. Soc. 311 (1989), no. 1, 185-208. MR 90a:11064

[4] M. Coppens: A study of the schemes $W_{e}^{1}$ of smooth plane curves, in Proc. 1st Belgian-Spanish Week on Algebra and Geometry, R.U.C.A (1988), 29-63.

[5] O. Debarre and M. Klassen: Points of low degree on smooth plane curves, J. Reine Angew. Math. 446 (1994), 81-87. MR 95f:14052

[6] D. Faddeev: On the divisor class groups of some algebraic curves, Soviet Math. Dokl. 2 (1961), 67-69. MR 24:A723

[7] G. Faltings: Diophantine approximation on abelian varieties, Ann. Math. 133 (1991), 549576. MR 93d:11066

[8] B. Gross and D. Rohrlich: Some results on the Mordell-Weil group of the Jacobian of the Fermat Curve, Invent. Math. 44 (1978), 201-224. MR 58:10911

[9] A. Hurwitz: Über die diophantische Gleichung $x^{3} y+y^{3}+x=0$, Math. Ann. 65 (1908), 428-430.

[10] M. Klassen and P. Tzermias: Algebraic points of low degree on the Fermat quintic, Acta Arith. 82 (1997), no. 4, 393-401. MR 98k:11086 
[11] F. Klein: Über die Tranformation siebenter Ordhang der elliptischen Funktionen, Gesammelte Math. Abhandlungen III 84, Springer, Berlin, 1923.

[12] N. Koblitz and D. Rohrlich: Simple factors in the Jacobian of a Fermat curve, Canadian J. Math., 30 (1978), no. 6, 1183-1205. MR 80d:14022

[13] S. Lefschetz: A Class of Algebraic Curves with Cyclic Group and their Jacobian Varieties, 163-178, in Selected Papers, Chelsea, New York, 1971. MR 45:8495

[14] C.-H. Lim: The Jacobian of a cyclic quotient of a Fermat curve, Nagoya Math. J. 125 (1992), 73-92. MR 93i:14024

[15] W. McCallum: On the Shafarevich-Tate group of the Jacobian of a quotient of the Fermat curve, Invent. Math. 93 (1988), no. 3, 637-666. MR 90b:11059

[16] D. Prapavessi: On the Jacobian of the Klein curve, Proc. Amer. Math. Soc. 122 (1994), no. 4, 971-978. MR 95b:14023

[17] P. Ribenboim: Homework!, Proc. 5th Conf. Canad. Number Th. Assoc., Ottawa (1996), 391-392, Amer. Math. Soc., Providence (1999).

[18] R. Taylor and A. Wiles: Ring-theoretic properties of certain Hecke algebras, Ann. Math. 141 (1995), no. 3, 553-572. MR 96d:11072

[19] P. Tzermias: Algebraic points of low degree on the Fermat curve of degree seven, Manuscripta Math. 97 (1998), 483-488. MR 99j:11075

[20] P. Tzermias: Parametrization of low-degree points on a Fermat curve, submitted for publication.

[21] A. Wiles: Modular elliptic curves and Fermat's last theorem, Ann. Math. 141 (1995), no. 3, 443-551. MR 96d:11071

Department of Mathematics, University of Tennessee, Knoxville, Tennessee 379961300

E-mail address: tzermias@math.utk.edu 